\title{
Left Dorsal Wall Anastomosis Compared with Parallel-Line Anastomosis for Intracorporeal Overlap Esophagojejunostomy during Laparoscopic Total Gastrectomy
}

\author{
Ji-Eun Lee, M.D., Ki Hyun Kim, M.D., Kyung Won Seo, M.D., Ph.D. \\ Department of Surgery, Kosin University College of Medicine, Busan, Korea
}

Purpose: This study evaluated the safety and efficacy of totally laparoscopic total gastrectomy (TLTG) with esophagojejunostomy by comparing left dorsal wall anastomosis (LDA) with parallelline anastomosis (PA) methods.

Methods: We retrospectively reviewed 28 patients who underwent laparoscopic total gastrectomy (LTG) for gastric cancer from January 2017 to September 2018. The patients were divided into two groups according to type of operation: 15 patients underwent PA and 13 underwent LDA. Clinicopathologic characteristics and surgical outcomes in the two groups were compared and analyzed.

Results: Surgical outcomes of estimated blood loss, operation time, and length of postoperative stay were similar between the two groups. In addition, there was no statistical difference in total operation time $(186.5 \pm 37.0$ min vs. $209.0 \pm 36.9$ min, $p=0.121)$ between the two groups. However, compared with the PA group, the LDA group required fewer additional stitches for reinforcement $(p=0.002)$.

Conclusion: Intracorporeal reconstruction of esophagojejunostomy using an LDA method may be a feasible and easy technique for TLTG compared with the PA method. Additional studies with larger sample sizes are needed to further support these results.

Keywords: Gastric neoplasms, Laparoscopy, Gastrectomy, Anastomosis
Received August 16, 2019

Revised October 1, 2019

Accepted October 14, 2019

Corresponding author

Kyung Won Seo

Department of Surgery, Kosin

University College of Medicine, 262

Gamcheon-ro, Seo-gu, Busan 49267, Korea

Tel: $+82-51-990-6782$

Fax: +82-51-246-6093

E-mail: kwseo@kosin.ac.kr ORCID:

https://orcid.org/0000-0002-5771-3832

This is an Open Access article distributed under the terms of the Creative Commons Attribution Non-Commercial License (http:// creativecommons.org/licenses/by-nc/4.0/) which permits unrestricted non-commercial use, distribution, and reproduction in any medium, provided the original work is properly cited.

Copyright @ 2020 The Journal of Minimally Invasive Surgery. All rights reserved.

\section{INTRODUCTION}

Gastric cancer is the fifth most common malignant tumor worldwide and ranks third among cancer deaths. ${ }^{1}$ Curative gastrectomy with lymph node dissection is the most recommended treatment for gastric cancer., ${ }^{2,3}$ With development of laparoscopic surgical instruments and techniques, laparoscopic gastrectomy has become widespread, and it is now possible to use laparoscopic procedures for all radical gastrectomy procedures, including laparoscopic total gastrectomy (LTG) and laparoscopic distal gastrectomy $(\mathrm{LDG}) .^{4-9}$
LTG is one of the most technically difficult methods for laparoscopic gastric cancer surgery. This is because, proper resection of lymph nodes is difficult, and intracorporeal esophagojejunostomy has been technically challenging for many years. However, numerous surgeons and researchers have contributed to developing different methods for intracorporeal esophagojejunostomy. ${ }^{10-15}$ Specifically, introduction of a V-roc suture known as a barbed suture and development of a stapler for use in laparoscopic surgery marked a turning point in laparoscopic surgery. In 2016, a study published by Lee SW and his colleagues found that the safety of intracorporeal 
anastomosis was improved during laparoscopic surgery when using a linear stapler and barbed suture..$^{16-18}$

Use of a barbed suture for entry hole closure after EJS using a linear stapler represents a great improvement; however, the pros and cons depending on angle of the stapler line are currently unknown. Thus, we compared the postoperative outcomes of parallel-lined anastomosis (PA) and left dorsal wall anastomosis (LDA) during esophagojejunostomy.

\section{MATERIALS AND METHODS}

From January 2017 to September 2018, 28 patients who underwent LTG to treat gastric cancer were included in this study. The patients were divided into two groups: Fifteen patients underwent PA and 13 patients underwent LDA. The criterion for total gastrectomy was proximal early gastric cancer, and LTG was attempted for early gastric cancer in patients that did not have confirmed perigastric LN metastasis (cNO).

During the procedure, the surgeon stood on the patient's right side, with the assistant and the endoscopist on the patient's left. A pneumoperitoneum $(12 \mathrm{mmHg})$ was established through an umbilical trocar, and the umbilical optical port served as a reference point for insertion of ports on either side of the patient's abdomen. The left lobe of the liver was retracted using a penrose catheter and nylone 2-0 to create the surgical field. Greater curvature mobilization was performed by dividing the gastrocolic ligament at least $3 \mathrm{~cm}$ from the gastroepiploic arcade. The left gastroepiploic vessels were divided, and dissection was continued in the same plane, proximally dividing the gastrosplenic ligament and controlling the short gastric vessels. This was continued until the superior pole of the spleen was completely free from the fundus. Distal mobilization up to the duodenum and the right gastroepiploic vessels was then performed. After duodenal transection, the right and left gastric vessels were divided, and medial-to lateral mobilization of the upper part of the stomach from the retroperitoneum was performed before esophageal transection. Lymph node dissection was performed during vascular mobilization according to the guidelines, removing the lymph nodes en-bloc on the side of resection. Vessels were ligated at their roots in most instances. Esophageal transection was then performed. There are different methods for performing PA and LDA; PA involves stapling with an intact esophagus and stomach. In LDA, the upper fundus area is held with the operator's left hand forceps and lifted so that the esophagus can be twisted about 60 degrees before stapling. Neither method staples the entire esophagus, leaving about $1 / 4$ of the organ to allow entry of the anvil side of the linear stapler during esophagojejunostomy. Next, the specimen is retrieved from the abdominal cavity for reconstruction.

The method of intracorporeal Roux-en-Y anastomosis after LTG is as follows: first, a linear stapler is used to cross the jejunum about $40 \mathrm{~cm}$ below Treitz's ligament. In the PA method, isoperistaltic side-to-side anastomosis is performed using a 45 mm linear stapler on the left side of the esophagus. Entry hole closure is initiated from the bottom using absorbable barbed sutures and closed by suturing the sero-muscular layer upward (Fig. 1A). In the LDA method, staplers are placed on the left dorsal wall of the esophagus, followed by side-to-side anastomosis with the jejunum (Fig. 1B, 2).
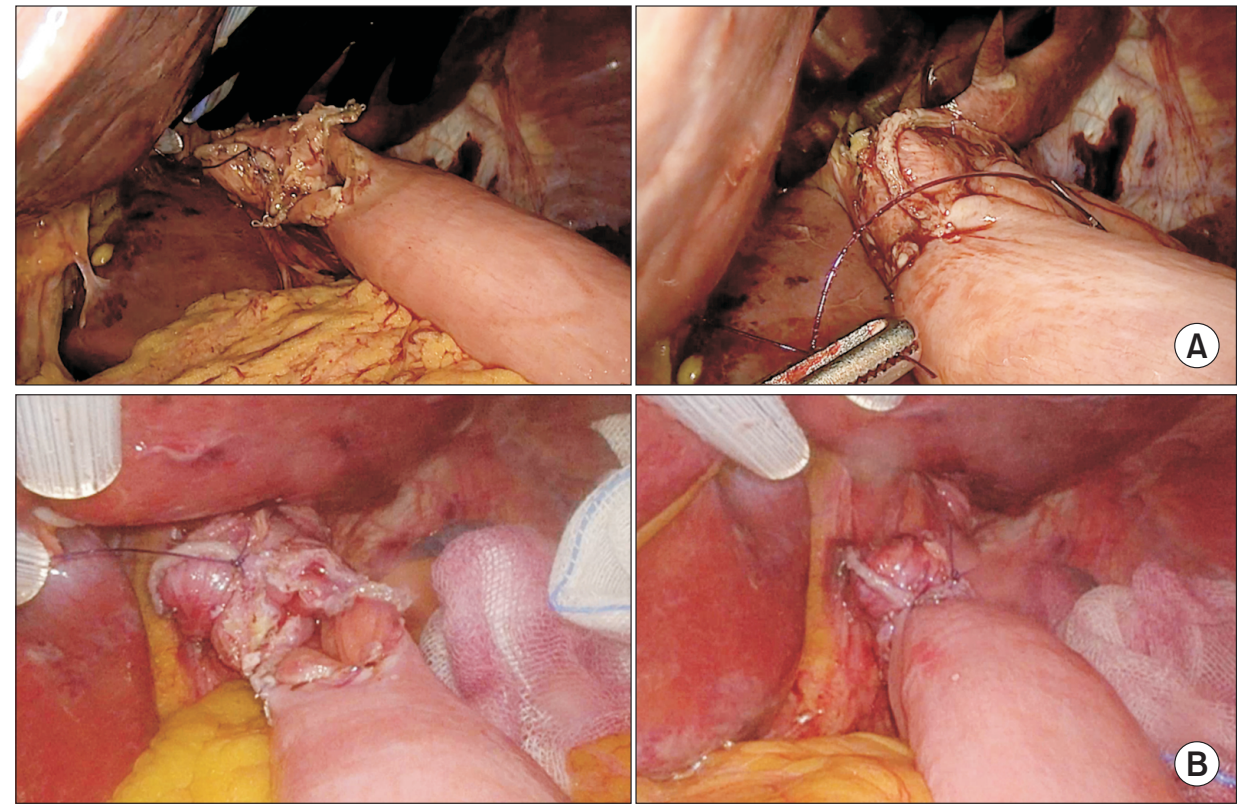

Fig. 1. (A) Conventional anastomosis. There was a challenge to orient the entry hole to make the suture easier. During the suture, it was difficult to hold the thread or organ with the instrument on the left hand to maintain the orientation. And the actual anastomotic direction was parallel. (B) LDA method. It is easy to suture the entry hole without turning the left hand. The enterotomy hole is exposed toward the surgeon. The actual anastomotic direction is directed to the left dorsal of the esophagus. 


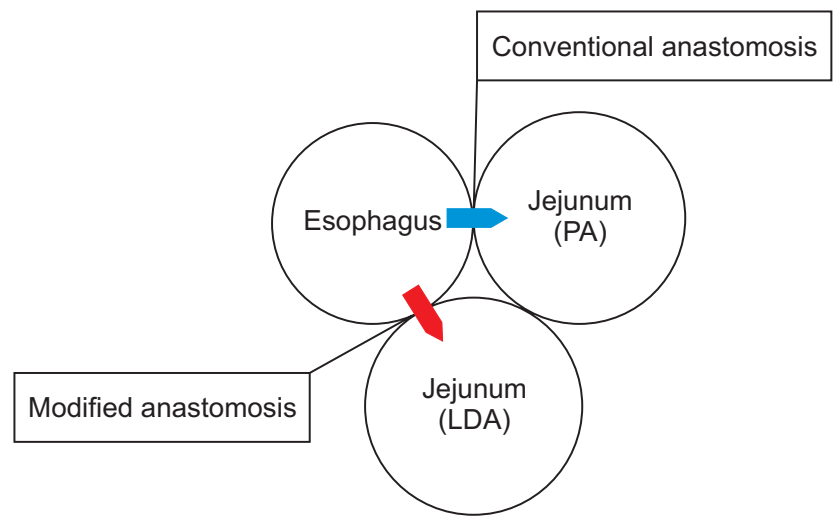

Fig. 2. Post-surgery schematic cross-sectional diagram.

Next, intracorporeal jejunojejunal anastomosis is performed. An enterotomy is made about $40 \mathrm{~cm}$ below the esophagojejunostomy, and a side-to-side jejunojejunostomy is made using a linear stapler on the jejunal stump on the Treitz's ligament side. The remaining enterotomy is sutured using a 3-0 V-Loc suture.

Finally, Petersen's and jejunojejunostomy mesenteric defects are closed by nonabsorbable (Ethibond 2-0) continuous $\mathrm{su}^{-}$ tures.

This study was approved by the Institutional Review Board of Kosin University Gospel Hospital, and informed consent was obtained from all patients before surgery.

All of the statistical analyses were performed using the Statistical Package for the Social Sciences (SPSS), version 22.0 for Windows (SPSS Inc, Chicago, USA). Categorical variables were analyzed by the Chi-square test or Fisher's exact test, while continuous variables were analyzed by Student's t test. The level of significance was set at $p<0.05$.

\section{RESULTS}

Clinicopathologic characteristics of patients are summarized in Table 1. Sex and dissection level were not significantly different in the PA and LDA groups. The postoperative pathology levels of the PA group indicated that 13 (86.7\%) and 2 patients (13.3\%) were in stage IA and stage IB, respectively. In the LDA group, there were 13 patients (100.0\%) and 0 patients in stage IA and stage IB, respectively. The mean age was 63.9 years for the PA group and 57.2 years for the LDA group, with a $p$ value of 0.037 .

There was no significant difference between the two groups in terms of postoperative stay or estimated blood loss $(p=0.809$, 0.335). Total operation time was also not significantly different between the two groups (186.5 $\pm 37.0 \mathrm{~min}$ vs. $209.0 \pm 36.9 \mathrm{~min}$, $p=0.121$. However, the number of reinforcement stitches re-
Table 1. Clinicopathologic characteristics of two types of esophagojejunostomy

\begin{tabular}{|c|c|c|c|}
\hline & $P A(N=15)$ & $\operatorname{LDA}(N=13)$ & $p$ value \\
\hline Age & $63.9 \pm 7.2$ & $57.2 \pm 8.9$ & 0.037 \\
\hline Sex & & & 1.000 \\
\hline M & $10(66.7 \%)$ & $8(61.5 \%)$ & \\
\hline $\mathrm{F}$ & $5(33.3 \%)$ & $5(38.5 \%)$ & \\
\hline BMl & $24.6 \pm 3.1$ & $23.4 \pm 3.5$ & 0.366 \\
\hline ASA & & & 0.512 \\
\hline Score 1 & $5(33.3 \%)$ & $7(53.8 \%)$ & \\
\hline Score 2 & $9(60.0 \%)$ & $5(38.5 \%)$ & \\
\hline Score 3 & $1(6.7 \%)$ & $1(7.7 \%)$ & \\
\hline Dissection level & & & 1.000 \\
\hline $\mathrm{D} 1+$ & $13(86.7 \%)$ & $11(84.6 \%)$ & \\
\hline $\mathrm{D} 2$ & $2(13.3 \%)$ & $2(15.4 \%)$ & \\
\hline Pathology (WHO class) & & & 0.578 \\
\hline WD tub & $4(26.7 \%)$ & $3(23.1 \%)$ & \\
\hline MD tub & $4(26.7 \%)$ & $2(15.4 \%)$ & \\
\hline PD tub & $3(20.0 \%)$ & $2(15.4 \%)$ & \\
\hline SRC & $4(26.7 \%)$ & $4(30.8 \%)$ & \\
\hline Cohesive type & $0(0.0 \%)$ & $2(15.4 \%)$ & \\
\hline Lauren & & & 0.068 \\
\hline Intestinal & $10(66.7 \%)$ & $6(46.2 \%)$ & \\
\hline Diffuse & $5(33.3 \%)$ & $3(23.1 \%)$ & \\
\hline Mixed & $0(0.0 \%)$ & $4(30.8 \%)$ & \\
\hline PRM_cm & $5.1 \pm 5.1$ & $4.9 \pm 3.4$ & 0.885 \\
\hline DRM_cm & $11.6 \pm 4.7$ & $11.7 \pm 3.6$ & 0.986 \\
\hline Pathologic stage (AJCC 7th) & & & 0.528 \\
\hline Stage IA & $13(86.7 \%)$ & $13(100.0 \%)$ & \\
\hline Stage IB & $2(13.3 \%)$ & $0(0.0 \%)$ & \\
\hline
\end{tabular}

$\mathrm{PA}=$ parallel-line anastomosis; $\mathrm{LDA}=$ left dorsal wall anastomosis; $\mathrm{BMI}=$ body mass index; $A S A=$ American society for anesthesiology; $W H O=$ World Health Organization; $W D=$ well-differentiated; $M D=$ moderately differentiated; $\mathrm{PD}=$ poorly differentiated; $\mathrm{SRC}=$ signet ring cell type; $\mathrm{PRM}=$ proximal resec tion margin; DRM = distal resection margin.

quired by the LDA group was lower than that of the PA group $(p=0.002)$ (Table 2).

\section{DISCUSSION}

The purpose of this study was to determine if an opera- 
Table 2. Surgical outcomes

\begin{tabular}{lccc}
\hline & PA (N=15) & LDA (N=13) $p$ value \\
\hline EBL (ml) & & \multicolumn{3}{c}{0.335} \\
$<20$ & $6(40.0 \%)$ & $8(61.5 \%)$ & \\
$20 \sim 50$ & $6(40.0 \%)$ & $4(30.8 \%)$ & \\
$50 \sim 100$ & $2(13.3 \%)$ & $0(0.0 \%)$ & \\
$100 \sim 150$ & $0(0.0 \%)$ & $1(7.7 \%)$ & \\
$150 \sim 300$ & $1(6.7 \%)$ & $0(0.0 \%)$ & \\
Operation time (min.) & $209.0 \pm 36.9$ & $186.5 \pm 37.0$ & 0.121 \\
Length of postoperative stay (days) & $8.9 \pm 2.7$ & $8.5 \pm 4.3$ & 0.809 \\
Additional suture (point) & & & 0.002 \\
0 & $0(0.0 \%)$ & $3(23.1 \%)$ & \\
1 & $2(13.3 \%)$ & $8(61.5 \%)$ & \\
2 & $8(53.3 \%)$ & $2(15.4 \%)$ & \\
3 & $5(33.3 \%)$ & $0(0.0 \%)$ & \\
\hline
\end{tabular}

$E B L=$ estimated blood loss.

tion procedure could be improved and made easier by adding a technical complement to the esophagojejunostomy method using a barbed suture and linear stapler, which are already widely used in laparoscopic surgery. In this study, we compared the esophagojejunal anastomosis method after LTG and found that the LDA group required fewer reinforced stitches than the PA group.

Among the laparoscopic gastric cancer approaches, LTG can be a burden even for surgeons with years of experience in laparoscopic surgery. This is due to insufficient resection of the lymph nodes and technical difficulties with intracorporeal esophagojejunostomy. Specifically, for intracorporeal suture and knot tying, the process can be difficult and timeconsuming, and the development has been slower than that of LDG. However, thanks to the efforts of many researchers, a variety of safe methods have been developed and are now practiced by many surgeons.

For example, whole hand-sewn esophagojejunostomy is rarely performed due to the technical difficulties with the procedure, which primarily use a circular or a linear stapler. The circular stapler does not need to be an additional suture for closing the inlet hole after the esophagojejunostomy, but it is necessary to expand the incision required to enter the circular stapler. The linear stapler can be performed immediately without extending the incision, but the challenge of entry hole closure is problematic. As closure of the stapler is a concern for food passage and requires detailed attention, many practitioners have considered hand-sewing the entry hole closure.
A paper published by Lee SW and his colleagues in 2016 introduced knotless barbed absorbable sutures and a suture entry hole after intracorporeal gastrointestinal anastomosis using a linear stapler. That study demonstrated the safety of this method by emphasizing that there were no complications such as leakage or stricture at the anastomosis site after surgery. ${ }^{18}$ Later, in other work in 2018, Lee SW studied how to suture entry holes using a linear stapler and two barbed sutures during esophagojejunostomy after LTG. In this retrospective study of 27 patients, there were no intraoperative complications such as gastrointestinal tears or injuries, and no postoperative leakage or stricture at the anastomosis site. ${ }^{19}$ Therefore, if the burden on the laparoscopic suture can be reduced, a crossover technique that is safe and employs a simple method is recommended.

Even with the same entry hole closure, we proposed this method to overcome the disadvantage caused by the parallel method, where the suture is started at the lower end surface of the entry hole farther from the operator's field of view. When the anastomosis was performed at 60 degrees on the dorsal side, the entire suture surface of the entry hole was brought into one field, and the suture could be easily started from the lower end, which resulted in fewer additional stitches. These results are likely attributable to the learning curve that occurs with development and familiarity with continuous operation of a single surgeon. However, considering that the previous 50 cases had used the PA method, the advantages of the LDA method seen in this study can be fully considered.

There were some limitations to this study. First, the study had a retrospective design. Second, the number of subjects was small $(n=28)$, so future studies should include a larger patient population to further examine these results. Third, since the results are based on procedures performed at a single institution by one operator, it is difficult to objectively determine if the results are generalizable for all operators that apply the LDA method. Additional studies should include multiple operators and be conducted at multiple hospital centers to better understand the impacts of individual operators and outcomes.

In conclusion, intracorporeal reconstruction of esophagojejunostomy using an LDA method is a feasible and easy technique for TLTG compared with the PA method. Based on the results of this study, operators can use this approach to achieve improved outcomes. Additional studies with larger sample populations are needed to confirm the results to improve guidelines for patient treatments.

\section{ORCID}

Ji-Eun Lee, https://orcid.org/0000-0003-4514-860X

Ki Hyun Kim, https://orcid.org/0000-0002-8296-3307 
Kyung Won Seo, https://orcid.org/0000-0002-5771-3832

\section{AUTHORS' CONTRIBUTIONS}

Study conception, design, and supervision: Ji-Eun Lee, Ki Hyun Kim, Kyung Won Seo; data collection, and data analysis and interpretation: Ji-Eun Lee, Ki Hyun Kim, Kyung Won Seo; writing of the first draft: Ji-Eun Lee, Ki Hyun Kim, Kyung Won Seo; review of medical records, data collection, and data analysis and interpretation: Ji-Eun Lee, Ki Hyun Kim, Kyung Won Seo; review of statistic data: Ji-Eun Lee, Ki Hyun Kim, Kyung Won Seo; and all authors were involved in critical revision of the paper.

\section{CONFLICT OF INTEREST}

None.

\section{FUNDING}

None.

\section{ACKNOWLEDGMENTS}

None.

\section{REFERENCES}

1) Ferlay J, Soerjomataram I, Dikshit R, et al. Cancer incidence and mortality worldwide: sources, methods and major patterns in GLOBOCAN 2012. Int J Cancer 2015;136:E359-386.

2) Sano T, Sasako M, Yamamoto $S$, et al. Gastric cancer surgery: morbidity and mortality results from a prospective randomized controlled trial comparing D2 and extended para-aortic lymphadenectomy--Japan Clinical Oncology Group study 9501. J Clin Oncol 2004;22:2767-2773.

3) Songun I, Putter H, Kranenbarg EM, Sasako M, van de Velde CJ. Surgical treatment of gastric cancer: 15-year follow-up results of the randomised nationwide Dutch D1D2 trial. Lancet Oncol 2010; 11:439-449.

4) Song KY, Park CH, Kang HC, et al. Is totally laparoscopic gastrectomy less invasive than laparoscopy-assisted gastrectomy?: prospective, multicenter study. J Gastrointest Surg 2008;12:10151021.

5) Kim JJ, Song KY, Chin HM, et al. Totally laparoscopic gastrectomy with various types of intracorporeal anastomosis using laparoscopic linear staplers: preliminary experience. Surg Endosc 2008; 22:436-442.

6) Ikeda O, Sakaguchi Y, Aoki Y, et al. Advantages of totally lapa- roscopic distal gastrectomy over laparoscopically assisted distal gastrectomy for gastric cancer. Surg Endosc 2009;23:2374-2379.

7) Lee SW, Bouras G, Nomura E, et al. Intracorporeal stapled anastomosis following laparoscopic segmental gastrectomy for gastric cancer: technical report and surgical outcomes. Surg Endosc 2010; 24:1774-1780.

8) Lee SW, Tanigawa N, Nomura E, et al. Benefits of intracorporeal gastrointestinal anastomosis following laparoscopic distal gastrectomy. World J Surg Oncol 2012;10:267.

9) Okabe H, Obama K, Tsunoda S, Tanaka E, Sakai Y. Advantage of completely laparoscopic gastrectomy with linear stapled reconstruction: a long-term follow-up study. Ann Surg 2014;259:109116.

10) Jung DH, Kim H-H. Laparoscopic Total Gastrectomy for Gastric Cancer. J Minim Invasive Surg 2015;18:31-38.

11) Shim JH, Yoo HM, Oh SI, et al. Various types of intracorporeal esophagojejunostomy after laparoscopic total gastrectomy for gastric cancer. Gastric Cancer 2013;16:420-427.

12) Chen K, Pan Y, Cai JQ, et al. Intracorporeal esophagojejunostomy after totally laparoscopic total gastrectomy: A single-center 7-year experience. World J Gastroenterol 2016;22:3432-3440.

13) Nagai E, Ohuchida K, Nakata K, et al. Feasibility and safety of intracorporeal esophagojejunostomy after laparoscopic total gastrectomy: inverted T-shaped anastomosis using linear staplers. Surgery 2013;153:732-738.

14) Son SY, Cui LH, Shin HJ, et al. Modified overlap method using knotless barbed sutures (MOBS) for intracorporeal esophagojejunostomy after totally laparoscopic gastrectomy. Surg Endosc 2017; 31:2697-2704.

15) Umemura A, Koeda K, Sasaki A, et al. Totally laparoscopic total gastrectomy for gastric cancer: literature review and comparison of the procedure of esophagojejunostomy. Asian J Surg 2015;38:102112.

16) Lee SW, Nomura E, Tokuhara T, et al. Laparoscopic technique and initial experience with knotless, unidirectional barbed suture closure for staple-conserving, delta-shaped gastroduodenostomy after distal gastrectomy. J Am Coll Surg 2011;213:e39-45.

17) Facy O, De Blasi V, Goergen M, Arru L, De Magistris L, Azagra JS. Laparoscopic gastrointestinal anastomoses using knotless barbed sutures are safe and reproducible: a single-center experience with 201 patients. Surg Endosc 2013;27:3841-3845.

18) Lee SW, Kawai M, Tashiro K, et al. Laparoscopic gastrointestinal anastomoses using knotless barbed absorbable sutures are safe and reproducible: a single-center experience with 242 patients. Jpn J Clin Oncol 2016;46:329-335.

19) Lee SW, Kawai M, Tashiro K, et al. The crossover technique for intracorporeal esophagojejunostomy following laparoscopic total gastrectomy: a simple and safe technique using a linear stapler and two barbed sutures. Surg Endosc 2019;33:1386-1393. 